\title{
Introducing molecular testing of pyrazinamide susceptibility improves multidrug-resistant tuberculosis treatment outcomes: a prospective cohort study
}

\author{
Feng Sun ${ }^{1,15}$, Yang $\mathrm{Li}^{1,15}$, Yu Chen ${ }^{2,15}$, Wenlong Guan ${ }^{3,15}$, Xiangao Jiang ${ }^{4,15}$, \\ Xiaomeng Wang ${ }^{5,15}$, Pengfei Ren ${ }^{2}$, Junlian $\mathrm{Li}^{3}$, Jichan $\mathrm{Shi}^{4}$, Guiqing $\mathrm{He}^{4}$, \\ Meiying $\mathrm{Wu}^{6}$, Peijun Tang ${ }^{6}$, Fei Wang ${ }^{5}$, Yunfeng Sheng ${ }^{7}$, Fuli Huang ${ }^{8}$, \\ Zumo Zhou ${ }^{9}$, Heqing Huang ${ }^{9}$, Liang Hong ${ }^{10}$, Qihui Liu ${ }^{1}$, Ying Zhang ${ }^{11}$ and \\ Wenhong Zhang ${ }^{1,12,13,14}$
}

@ERSpublications

Early availability of reliable PZA drug-susceptible testing is a prerequisite for successful MDR-TB treatment. The optimised regimen achieved a treatment success rate of $82.4 \%$ and shortened the course to 12 months for PZA-susceptible patients http://ow.ly/ObdI30n3GF7

Cite this article as: Sun F, Li Y, Chen Y, et al. Introducing molecular testing of pyrazinamide susceptibility improves multidrug-resistant tuberculosis treatment outcomes: a prospective cohort study. Eur Respir J 2019; 53: 1801770 [https://doi.org/10.1183/13993003.01770-2018].

ABSTRACT The current treatment for multidrug-resistant tuberculosis (MDR-TB) takes a lengthy period of $18-24$ months and has a poor cure rate of $50-60 \%$. A multicenter, prospective cohort study was conducted to assess the role of testing for molecular susceptibility to pyrazinamide (PZA) in optimising treatment for MDR-TB.

We assigned 76 patients to an optimised molecular susceptibility group and 159 patients to a regular treatment group where PZA susceptibility was not determined. Of these patients, 152 were matched after propensity score matching (76 in the optimised group and 76 in the regular group). Treatment success rate was measured in the propensity-matched cohort as the primary outcome.

Patients in the optimised group achieved a higher treatment success rate than those in the regular group (76.3\% versus $55.3 \%, \mathrm{p}=0.006$ ). Of 51 patients with isolates that were susceptible to PZA and who were receiving a 12-month regimen, $42(82.4 \%)$ were treated successfully. The optimised group showed faster culture conversion than the regular group $(\mathrm{p}=0.024)$. After exclusion of pre-extensively drug-resistant TB (pre-XDR-TB), the treatment outcome in the optimised group was still better than the regular group ( $83.1 \%$ versus $62.1 \%, \mathrm{p}=0.009$ ).

Introducing molecular susceptibility testing for PZA improved the treatment outcomes for MDR-TB without the use of new drugs. Introducing PZA for patients with PZA-susceptible (PZA-S) MDR-TB allows the current regimen to be shortened to 12 months with comparable success rates to the World Health Organization (WHO) recommended shorter regimen.

This article has supplementary material available from erj.ersjournals.com

This study is registered at https://clinicaltrials.gov with identifier NCT02120638. No additional unpublished data are available. 


\section{Introduction}

Multidrug-resistant tuberculosis (MDR-TB), defined as tuberculosis (TB) with resistance to isoniazid and rifampicin, significantly threatens TB control worldwide [1]. The treatment for MDR-TB generally requires 18-24 months of therapy with second-line agents, which are more toxic and less effective than first-line drugs. Treatment success rates for MDR-TB using the conventional World Health Organization (WHO) 20-month regimen are estimated to be about $54 \%$ worldwide, which is unacceptably low [1].

Recent studies have attempted to improve outcomes and shorten the course of MDR-TB treatment by adding new drugs or clearly identifying "simple MDR-TB" (i.e. MDR-TB that is only resistant to rifampicin and isoniazid, but which is susceptible to other second-line drugs). Based on successful experiences in Bangladesh and several African countries, the WHO has recommended a standardised shorter regimen for MDR-TB patients who are probably susceptible to second-line drugs (the Bangladesh regimen, 4-6 Km-Gfx-Pto-Cfz-PZA-H (high-dose)-EMB/5Gfx-Cfz-PZA-EMB) [2]. Furthermore, stage 2 of the STREAM trial is trying to shorten the course to 28 weeks by adding bedaquiline [3]. We noted that pyrazinamide (PZA) is included in the WHO shorter regimen, the STREAM trial and a dozen other MDR-TB clinical trials [4-6]. Regrettably, high rates of resistance to PZA are present in several countries [7, 8] where drug susceptibility testing (DST) to PZA is not mandatory for all patients. Given that the use of rapid molecular detection systems, such as Xpert-MTB/RIF, has been proven to lead to more prompt initiation of appropriate TB treatment [9], optimised background regimens based on rapid molecular DST information might be another useful strategy for improving MDR-TB outcomes and even for shortening treatment duration utilising the important sterilising activity of PZA [10]. Additionally, performance of molecular DST for fluoroquinolones has been recommended before selecting a shorter regimen, in order to ensure this key drug susceptibility and might be applied to guide the application of shortened MDR-TB regimens [11].

PZA is a key frontline anti-TB drug that shows unique sterilising activity that is critical for shortening TB therapy $[10,12,13]$. Recent studies have shown good penetration of PZA into lung tissues of MDR-TB patients with a significant correlation between the tissue concentration of PZA and necrosis [14]. In drug-susceptible TB, adding PZA to rifampicin and isoniazid has allowed the shortening of the duration of treatment from 9 months to 6 months [15]. As a pivotal drug for the treatment for patients with MDR-TB, PZA is recommended to be used routinely for the entire treatment duration unless there is confirmed resistance $[2,16]$. However, proper use of PZA in MDR-TB treatment has been confounded by PZA resistance, which varies from $10 \%$ to $85 \%$ in different geographic settings [17-19]. As such, inappropriate inclusion of PZA could amplify drug resistance or possibly cause unnecessary toxicity among patients with PZA-resistant (PZA-R) MDR-TB [20].

Appropriate evaluation of PZA resistance for MDR-TB thus appears to be a prerequisite for an effective regimen, both to improve the outcome or to shorten the treatment duration [21]. Although traditional DST for PZA is still technically challenging [22], sequencing of the pncA gene associated with PZA resistance [23] has been demonstrated to be both more sensitive and more specific than phenotype DST [10, 24] (which is prone to false resistance as well as to missing low-level PZA resistance caused by nonsynonymous mutations in pncA $[25,26])$. A series of studies have shown promising consistency between molecular detection of PZA resistance and traditional methods, providing a robust basis for this study [18, 27].

In an attempt to address the hypothesis that treatment for PZA-susceptible (PZA-S) MDR-TB may be shortened [21], this study evaluates the rapid molecular PZA-susceptibility-based MDR-TB treatment regimen in shortening treatment from 24 months to 12 months for PZA-S MDR-TB and improving the treatment outcomes for PZA-R MDR-TB patients.

Affiliations: ${ }^{1}$ Dept of Infectious Diseases, Huashan Hospital, Fudan University, Shanghai, China. ${ }^{2}$ Dept of Tuberculosis, Henan Province Infectious Diseases Hospital, Zhengzhou, China. ${ }^{3}$ The Chest Hospital of Xinjiang Uyghur Autonomous Region, Ürümqi, China. ${ }^{4}$ Dept of Infectious Diseases, Wenzhou Central Hospital, Wenzhou, China. ${ }^{5}$ Zhejiang Provincial Center for Disease Control and Prevention, Hangzhou, China. ${ }^{6}$ Suzhou Fifth People's Hospital, Suzhou, China. ${ }^{7}$ Tuberculosis Treatment Center, Hangzhou Red Cross Hospital, Hangzhou, China. ${ }^{8}$ Dept of Infectious Diseases, The Affiliated Hospital of Southwest Medical University, Luzhou, China. ${ }^{9}$ People's Hospital of Zhuji, Zhuji, China. ${ }^{10}$ Dept of Infectious Diseases, The Third Affiliated Hospital to Wenzhou Medical College, Rui'an, China. ${ }^{11}$ Dept of Molecular Microbiology and Immunology, Bloomberg School of Public Health, John Hopkins University, Baltimore, MD, USA. ${ }^{12}$ State Key Laboratory of Genetic Engineering, School of Life Sciences, Fudan University, Shanghai, China. ${ }^{13}$ National Clinical Research Center for Aging and Medicine, Huashan Hospital, Fudan University, Shanghai, China. ${ }^{14}$ Key Laboratory of Medical Molecular Virology $(\mathrm{MOE} / \mathrm{MOH})$ and Institute of Biomedical Sciences, Shanghai Medical College, Fudan University, Shanghai, China. ${ }^{15}$ These authors contributed equally to this work.

Correspondence: Wenhong Zhang, Room 501, Building 5, Huashan Hospital, No.12, Middle Wulumuqi Road, Jing'an District, Shanghai, China. E-mail: zhangwenhonglafudan.edu.cn 


\section{Methods}

\section{Study design and participants}

This phase 3, multi-center, prospective open-label study was launched in 2014 with the aim of optimising the treatment for MDR-TB based on the results of molecular susceptibility testing of PZA. 12 hospitals located in six provinces in China (Zhejiang, Jiangsu, Sichuan, Xinjiang, Henan and Chongqing) were selected to represent a diverse patient population. Patients aged between 18 years and 65 years with active laboratory-confirmed pulmonary MDR-TB were eligible for inclusion. Patients were excluded in cases of proven or probable extensively drug-resistant TB (XDR-TB), a Karnofsky performance status score of 50 or less, a history of active TB of more than 3 years, HIV and specific baseline laboratory abnormalities (supplementary material).

\section{Study procedures}

DST for isoniazid and rifampicin was performed from the first positive Mycobacterium tuberculosis culture using the proportion method. The patients of the optimised group were enroled from April 2014 to November 2014, after which time the patients of the regular group were enroled (December 2014 to August 2015). Participants in the regular group were assigned for a conventional 24-month regimen according to WHO recommendations, which included PZA, amikacin, levofloxacin, prothionamide and clarithromycin or cycloserine if cycloserine was available [28].

For participants in the optimised group additional DST for PZA was performed, through sequencing of $p n c A$ [23] and $r p s A$ [29], in order to tailor the treatment regimen appropriately. $p n c A$ was amplified with primers pncA-F (5'-GTCGGTCATGTTCGCGATCG-3') and pncA-R (5'-GCTTTGCGGCGAGCGCTCCA-3'). rpsA was amplified with primers rpsA-F (5'-GGCCGCAGCTGGGACGCGGC-3') and rpsA-R (5'CGGTCCAGCGCTCCGTCTGC-3'). In case of any discrepancy in results between phenotypic DST and molecular DST, molecular DST would prevail. PZA-S patients were treated with a 12-month regimen including an intensive phase of 6 months with PZA, amikacin, levofloxacin, clarithromycin and prothionamide and a consolidation phase of 6 months with PZA, levofloxacin, clarithromycin and prothionamide (6PZA-Amk-Lfx-Pto-Clr/6PZA-Lfx-Pto-Clr). For PZA-R patients, PZA was replaced by high-dose isoniazid and the regimen consisted of an intensive phase of 6 months and a continuation phase of 18 months (6Amk-Lfx-Pto-Clr- $\mathrm{H}^{\mathrm{h}} / 6 \mathrm{Lfx}-\mathrm{Pto}-\mathrm{Clr}-\mathrm{H}^{\mathrm{h}}$ ). The drug dosages used in this study are shown in table 1. Follow-ups were carried out monthly for the first 12 months and then every 2 months for 13-24 months. Follow-ups after cure continued for 1 year, with sputum smear and culture every 6 months. Safety evaluations were performed including the routine laboratory tests (blood, urine, liver function, kidney function and electrolytes), blood glucose tests, hearing tests, vital signs, ECGs and chest computed tomography (CT) scans. Adverse events were monitored and promptly managed during the entire treatment course. At the end of follow-up, two physicians who were blinded to the patients' regimen determined the treatment outcomes according to the definitions and reporting framework for TB recommended by the WHO in 2013 [30] and the study protocol, as the WHO reporting framework had not been published when this study was designed (supplementary table S1). Additionally, PZA DST was performed retrospectively in the regular cohort.

All patients gave written informed consent and the study protocol was approved by the ethics committee of Huashan Hospital affiliated to Fudan University. This study was registered on ClinicalTrials.gov, number NCT02120638.

TABLE 1 Treatment regimen composition and daily drug dosages by weight group

\begin{tabular}{|c|c|c|c|c|c|}
\hline \multirow[t]{2}{*}{ Drug } & \multicolumn{2}{|c|}{ Regimen } & \multicolumn{3}{|c|}{ Weight group ${ }^{\#}$} \\
\hline & Regular (n=76) & Optimised (n=76) & $33-50 \mathrm{~kg}$ & $51-70 \mathrm{~kg}$ & $>70 \mathrm{~kg}$ \\
\hline Pyrazinamide & $76(100)$ & 51 (67) & $1000-1750$ & $1750-2000$ & $2000-2500$ \\
\hline Isoniazid & $0(0)$ & $25(32)$ & 600 & 600 & 600 \\
\hline Amikacin & $76(100)$ & $76(100)$ & 600 & 600 & 600 \\
\hline Levofloxacin & $76(100)$ & $76(100)$ & 750 & 750 & 1000 \\
\hline Prothionamide & $76(100)$ & 76 (100) & 500 & 750 & 1000 \\
\hline Clarithromycin & 40 (53) & $76(100)$ & 500 & 1000 & 1000 \\
\hline Cycloserine $^{+}$ & $36(47)$ & $0(0)$ & 500 & 750 & 1000 \\
\hline
\end{tabular}

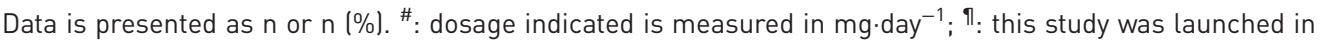
April 2014 following the 2011 updated World Health Organization (WHO) guidelines which allowed the use of clarithromycin; ${ }^{+}$: cycloserine was not introduced in China until the end of 2014 . Thus, 36 out of 76 patients in the regular regimen group received this drug when subsequently available. 


\section{Statistical analysis}

A propensity score approach was used to control for potential covariates that might influence the choice of whether to administer the optimised regimen. Propensity matching scores were estimated by the multivariate logistic regression model with the independent variables including age, presence of cavity and previous second-line drug treatment $[31,32]$. We matched the regular group and the optimised group one-to-one within a caliper of 0.2 standard deviations of the logit of the propensity score [33], without replacement. The primary analysis was based on propensity score matching.

\section{Results}

\section{Study participants}

Recruitment began in April 2014 and the last patient visit was completed in August 2017. A total of 316 patients with proven or suspected MDR-TB were assessed for eligibility (figure 1). Amongst these patients, 262 subjects aged between 18 years and 65 years were confirmed by laboratory testing to have MDR-TB, but 10 patients declined to participate in the study for personal reasons. 14 patients were excluded because

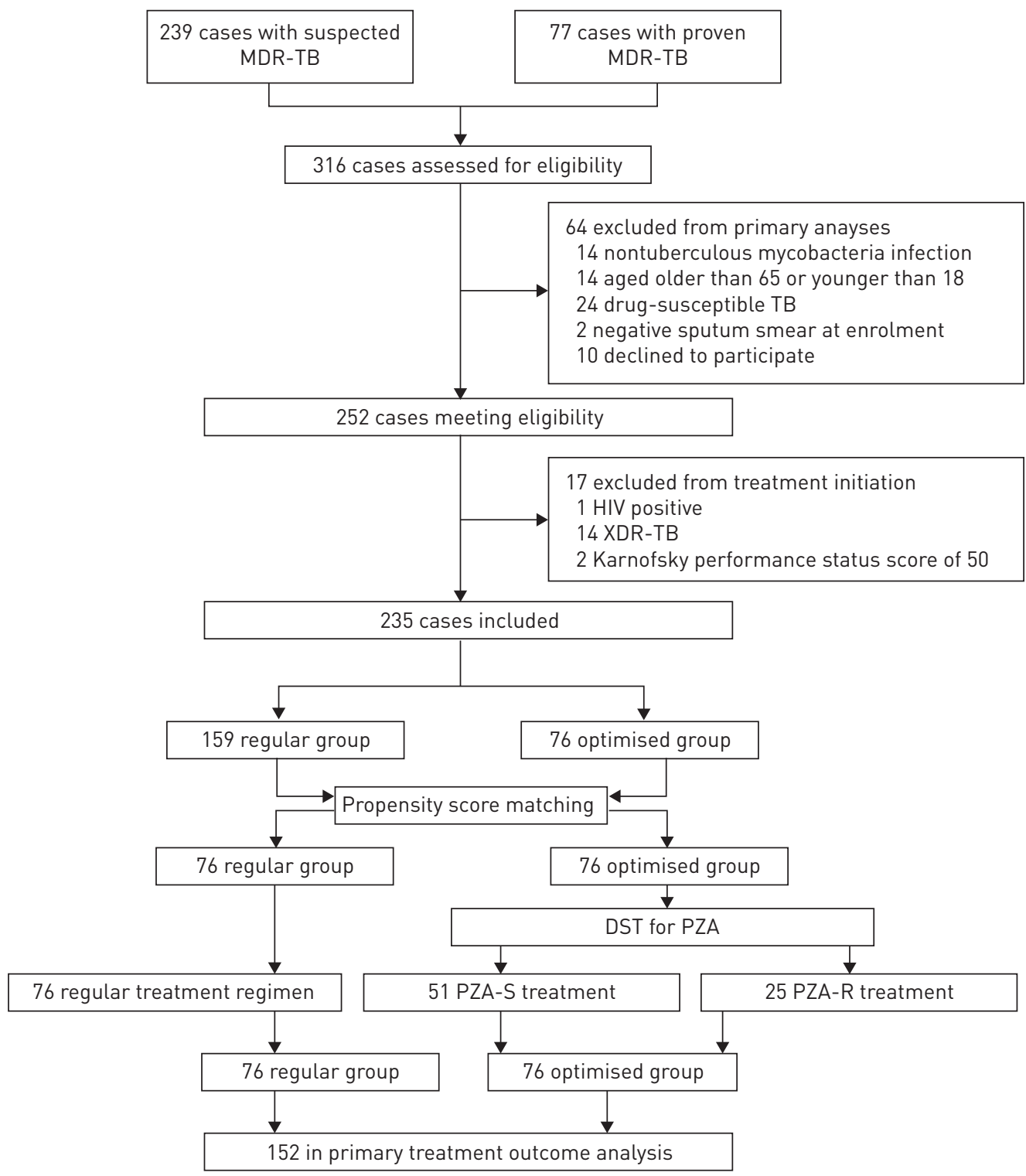

FIGURE 1 Flow chart of the study. Among the 316 patients assessed for eligibility, 235 cases with laboratory-confirmed multidrug-resistant TB (MDR-TB) were included (159 in the regular group and 76 in the optimised group). Of these cases, 152 were matched (76 in each group) and were included in the primary analysis. XDR-TB: extensively drug-resistant tuberculosis; DST: drug susceptibility testing; PZA: pyrazinamide; PZA-S: PZA-susceptible; PZA-R: PZA-resistant. 
of definite or suspected XDR-TB infection, while two were excluded due to a low Karnofsky performance status score. Finally, one patient with HIV was also excluded.

As a result, 235 cases with laboratory-confirmed MDR-TB were included (159 in the regular group and 76 in the optimised group). A propensity score was calculated for these 235 subjects with complete data and 152 of them were matched (76 in each group). Matched groups had an adequate balance of most baseline sociodemographic and clinical characteristics (table 2). Of the 152 patients assessed for treatment outcome, 104 were men (median age 36 years, interquartile range (IQR) 25-45). Approximately $85 \%$ of the patients had received anti-TB treatment before allocation and nearly $20 \%$ had been treated with fluoroquinolones or second-line injectable drugs (SLIDs). The 76 patients allocated to the optimised group were subjected to molecular DST for PZA by pncA and rpsA sequencing. Of these patients, 25 had isolates with molecular resistance to PZA, while the remaining 51 were susceptible to PZA.

\section{Treatment outcomes}

Patients in the optimised group achieved a higher treatment success rate than the regular group according to both the WHO definition ( $76.3 \%$ versus $55.3 \%, \mathrm{p}=0.006$; figure $2 \mathrm{a}$ ) and the study definition $(76.3 \%$ versus $57.9 \%, \mathrm{p}=0.016$; figure $2 \mathrm{~b})$. Notably, $42 \mathrm{PZA}-\mathrm{S}$ patients $(82.4 \%)$ receiving the shorter regimen were

\begin{tabular}{|c|c|c|c|}
\hline \multirow[t]{2}{*}{ Characteristic } & \multicolumn{2}{|c|}{ Regimen } & \multirow[t]{2}{*}{ p-value } \\
\hline & Regular (n=76) & Optimised (n=76) & \\
\hline Age years & & & 0.165 \\
\hline Mean & $37.1 \pm 12.3$ & $34.6 \pm 11.8$ & \\
\hline Median & $36.5(27-47)$ & $33.5(25-41)$ & \\
\hline Sex & & & 0.116 \\
\hline Male & $57(75.0)$ & 47 (61.8) & \\
\hline Female & 19 (25.0) & 29 (38.2) & \\
\hline BMI $\mathrm{kg} \cdot \mathrm{m}^{-2}$ & & & 0.320 \\
\hline Mean & $19.5 \pm 2.4$ & $19.8 \pm 2.5$ & \\
\hline Median & $19.4(18.0-20.8)$ & $19.5(18.4-21.2)$ & \\
\hline Occupation & & & 0.650 \\
\hline Farmer & 25 (32.9) & $26(34.2)$ & \\
\hline Labourer & $16(21.1)$ & $19(25.0)$ & \\
\hline Employed & $10(13.2)$ & $14(18.4)$ & \\
\hline Unemployed & $15(19.7)$ & $10(13.2)$ & \\
\hline Other & 10 (13.2) & $7(9.2)$ & \\
\hline \multicolumn{4}{|l|}{ TB symptoms } \\
\hline Cough & 67 (88.2) & $62(81.6)$ & 0.258 \\
\hline Expectoration & 58 (76.3) & $62(81.6)$ & 0.426 \\
\hline Haemoptysis & 33 (43.4) & $51(67.1)$ & 0.003 \\
\hline Fever & $17(22.4)$ & 34 (44.7) & 0.006 \\
\hline Chest pain & $9(11.8)$ & $8(10.5)$ & 0.797 \\
\hline Dyspnoea & $7(9.2)$ & $14(18.4)$ & 0.157 \\
\hline Fatigue & $15(19.7)$ & $23(30.3)$ & 0.134 \\
\hline \multicolumn{4}{|l|}{ Chest radiograph } \\
\hline Presence of cavity & $51(67.1)$ & 58 (76.3) & 0.280 \\
\hline Bilateral involvement & $58(75.0)$ & $61(80.3)$ & 0.776 \\
\hline Medical history of diabetes mellitus & $19(26.3)$ & $14(18.4)$ & 0.325 \\
\hline Any smoking history & $6(7.9)$ & $10(16.2)$ & 0.429 \\
\hline Alcohol consumption & $5(6.6)$ & $6(7.9)$ & 0.763 \\
\hline Previous second-line drugs & & & 0.819 \\
\hline Never treated & $59(77.6)$ & $62(81.6)$ & \\
\hline Treatment-naïve & $16(21.1)$ & $8(10.5)$ & \\
\hline First-line only & $43(56.6)$ & $54(71.1)$ & \\
\hline One second-line drug & $13(17.1)$ & $11(14.5)$ & \\
\hline Fluoroquinolones & $9(11.8)$ & $6(7.9)$ & \\
\hline Aminoglycosides & $4(5.3)$ & $5(6.6)$ & \\
\hline Two or more second-line drugs & $4(5.3)$ & $3(3.9)$ & \\
\hline
\end{tabular}

Data is reported as $\mathrm{n}(\%)$, mean $\pm \mathrm{SD}$ and median (IQR), unless otherwise stated. BMI: body mass index; TB: tuberculosis. 
a)
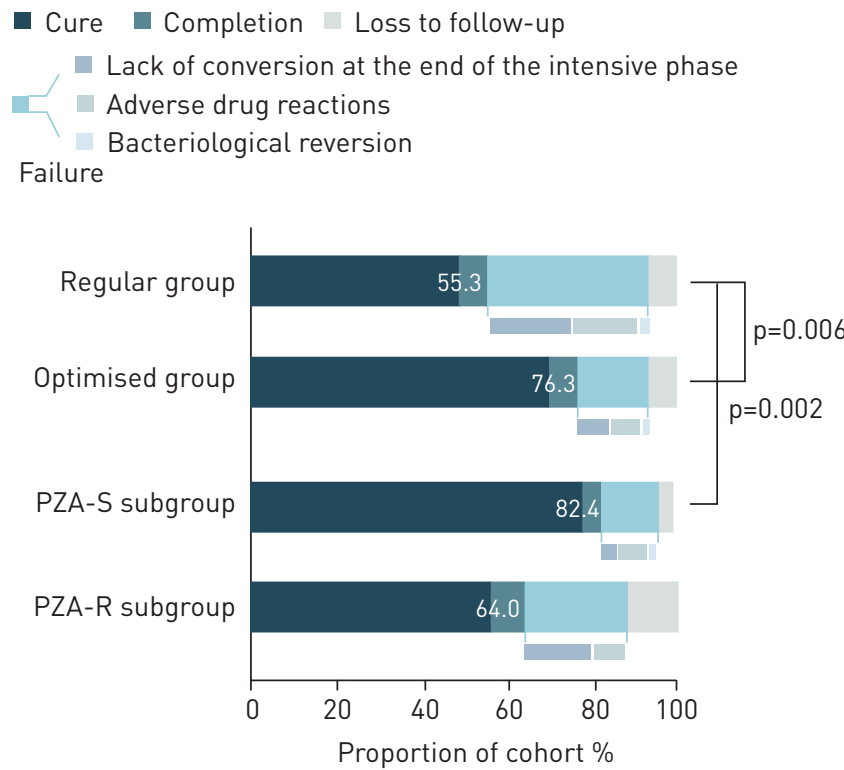

$\begin{array}{rcccc} & \text { Cure } & \text { Completion } & \text { Failure } & \text { follow-up } \\ \text { Regular group }(n=76) & 37(48.7) & 5(6.6) & 29(38.2) & 5(6.6) \\ \text { Optimised group }(n=76) & 54(71.1) & 4(5.3) & 13(17.1) & 5(6.6) \\ \text { PZA-S }(n=51) & 40(78.4) & 2(3.9) & 7(13.7) & 2(3.9) \\ \text { PZA-R }(n=25) & 14(56.0) & 2(8.0) & 6(24.0) & 3(12)\end{array}$

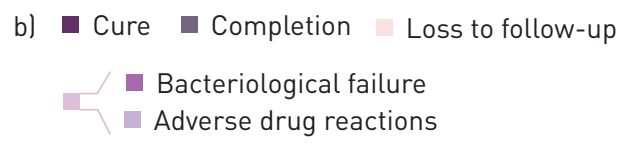

Failure

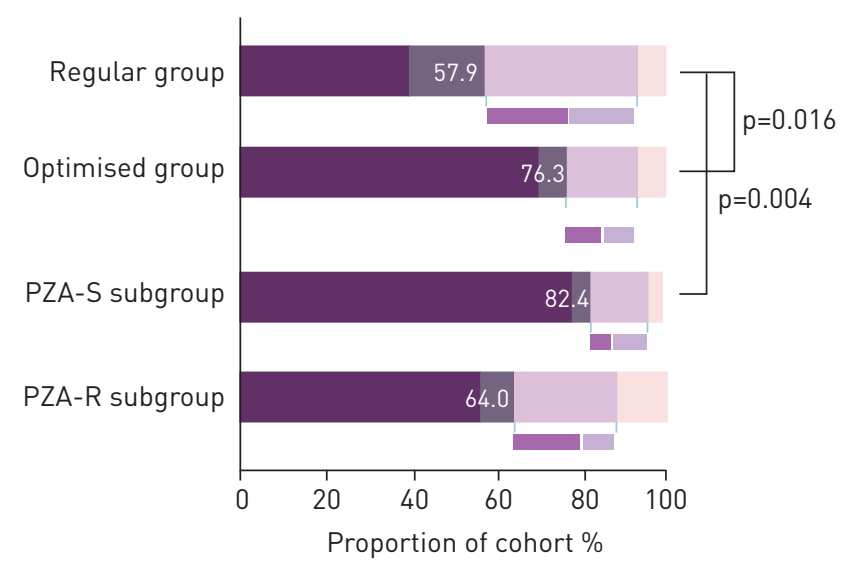

Loss to

Cure Completion Failure follow-up

Regular group $(n=76) \quad 30(39.5) \quad 14(18.4) \quad 27(35.5) \quad 5(6.6)$

Optimised group $(n=76) \quad 54(71.1) \quad 4(5.3) \quad 13(17.1) \quad 5(6.6)$

PZA-S $(n=51) \quad 41(80.4) \quad 1(2.0) \quad 7(13.7) \quad 2(3.9)$

PZA-R $(n=25) \quad 13(52.0) \quad 3(12.0) \quad 6(24.0) \quad 3(12)$

FIGURE 2 Overall treatment outcomes for multidrug-resistant tuberculosis grouped by regimen category according to an analysis based on World Health Organization definitions (a) and the protocol-defined analysis (b). Data in the bars are treatment success rates. Data below the graphics are presented as $\mathrm{n}(\%)$. Patients in the optimised group were classified into two subgroups, pyrazinamide-susceptible (PZA-S) and pyrazinamide-resistant (PZA-R).

treated successfully, showing a significantly greater improvement than the regular group $(27.1 \%, 95 \% \mathrm{CI}$ $10.1-43.4 \%$; $=0.002$ ). The causes of treatment failure amongst the two groups were slightly different. Absence of conversion at the end of the 6-month intensive phase represented the most common reason for treatment failure in the regular group, followed by adverse drug reactions and sputum reversion (relative responsibilities were $51.7 \%, 41.4 \%$ and $6.9 \%$, respectively). In contrast, of the seven patients with treatment failure in the PZA-S subgroup, four discontinued treatment due to adverse drug reactions, two did not have culture conversion within the intensive phase and one achieved subsequent reversion. The remaining two PZA-S patients were lost to follow-up during treatment and did not complete the course (in total, five patients in each treatment group were lost to follow-up in this fashion).

Of the 58 patients who achieved successful treatment outcomes in the optimised group, 35 out of 42 $(83.3 \%)$ in the PZA-S group and 13 out of $16(81.3 \%)$ in the PZA-R group completed post-treatment follow-up. Of these 48 patients, 44 provided sputum samples for smear and culture examination, and all were negative, while the other four patients were asymptomatic with a normal chest CT scan.

When compared with the regular regimen, the optimised regimen resulted in quicker sputum conversion with a hazard ratio of 0.66 ( $95 \%$ CI $0.45-0.94, p=0.024$; figure 3 ). The curves for culture conversion showed discernible differences in the proportion of intensive phase sputum-culture conversion between the optimised group and the regular group ( $86.8 \%$ versus $75 \%, \mathrm{p}=0.033)$. In the optimised group, 48 out of 51 patients $(94.2 \%)$ in the PZA-S subgroup and 20 out of 25 patients $(80 \%)$ in the PZA-R group had culture conversion at the end of the 6-month intensive phase $(\mathrm{p}=0.054)$.

\section{Secondary analysis}

A secondary analysis measured differences between two groups with respect to the treatment success rate after exclusion of pre-XDR-TB. A total of 123 patients were selected if they had not been treated with any second-line drug treatment or could be excluded from second-line drug resistance according to susceptibility testing. The overall success rate improved compared to the primary analysis and reached $86.2 \%$ in the PZA-S subgroup. The treatment outcome in the optimised group was still significantly better than in the regular group, with success rates of $83.1 \%$ and $62.1 \%$, respectively $(\mathrm{p}=0.009$, figure $4 \mathrm{a}$ ). Furthermore, a similar difference was observed amongst the 74 patients who were proven to be susceptible 
FIGURE 3 Proportion of patients with positive sputum cultures and time to conversion.

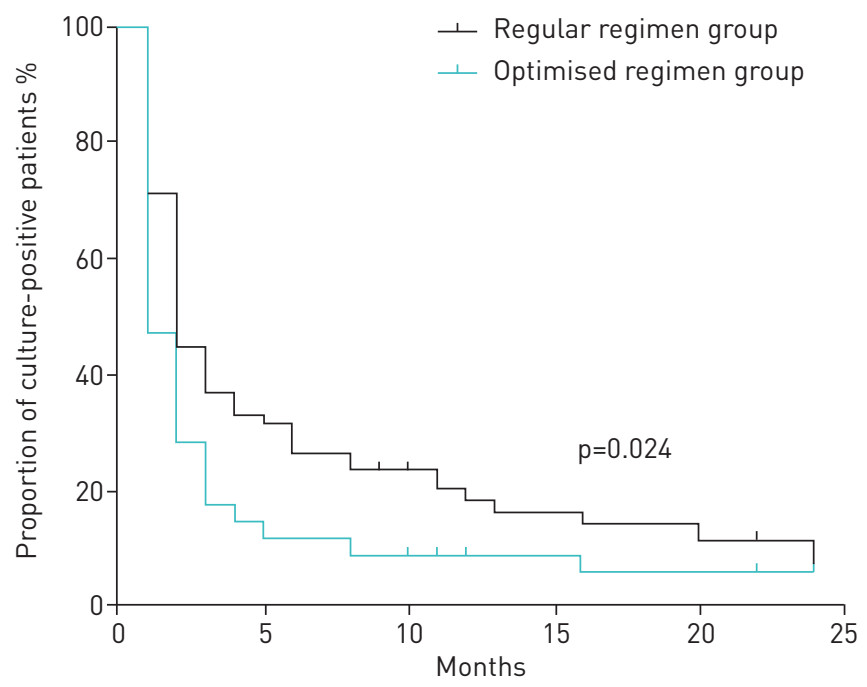

to all second-line drugs by available DST results (34 in the optimised group and 40 in the regular group). Finally, for those patients in the optimised group who experienced treatment failure, in no cases was this due to bacteriological failure (figure $4 \mathrm{~b}$ ).

\section{Subgroup analysis}

Retrospective molecular DST for PZA was performed amongst the regular group and identified 29 patients with PZA-S MDR-TB and 21 patients with PZA-R MDR-TB (the isolates from the remaining 26 patients were not available). For this analysis, higher success rates were found in the optimised group regardless of susceptibility to PZA, although without significance (figures $5 \mathrm{a}$ and $5 \mathrm{~b}$ ). Moreover, the time to conversion

a)

$$
\text { Failure }
$$

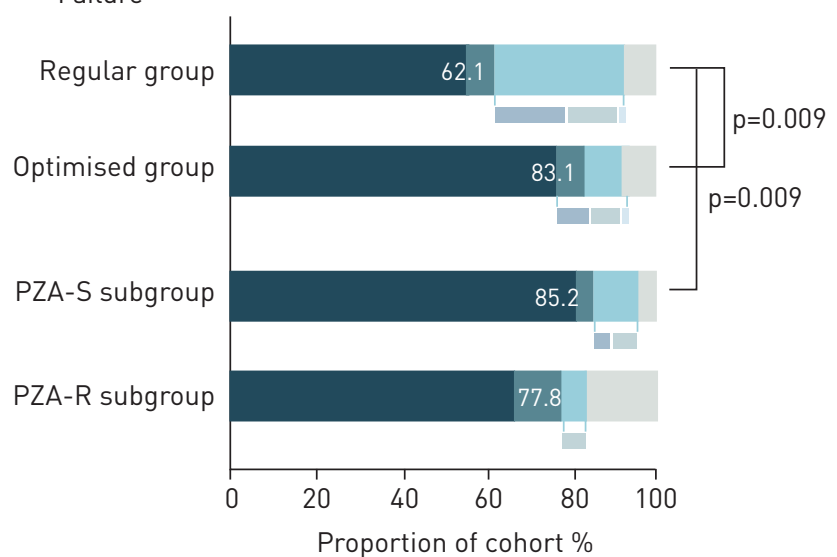

Proportion of cohort \%
Cure

Completion

$$
4 \text { (6.9) }
$$

$4(6.2)$

$2(4.3)$

$2(11.1)$

Loss to

Regular group ( $n=58$ )

Optimised group $(n=65)$

PZA-S (n=47)

PZA-R (n=18)
$50(76.9)$

$38(80.9)$

$12(66.7)$
Failure

$$
18 \text { (31) }
$$

4 (6.9)

6 (9.2)

$5(7.7)$

$5(13.7)$

$2(4.3)$

1 (5.6)

$3(16.7)$ follow-up b)

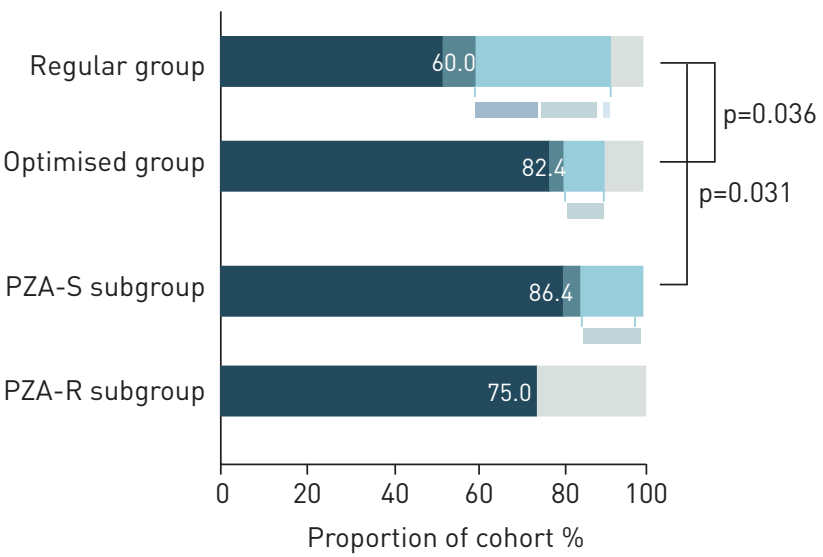

Proportion of cohort \%

Cure Completion Failure follow-up

Regular group $(n=40) \quad 21(52.5) \quad 3(7.5) \quad 13(32.5) \quad 3(7.5)$

Optimised group (n=34) $27(79.4) \quad 1(2.9) \quad 3(8.8) \quad 3(8.8)$

$\begin{array}{rrrr}27(79.4) & 1(2.9) & 3(8.8) & 3(8.8) \\ 18(81.8) & 1(4.5) & 3(13.6) & 0(0)\end{array}$

$9(75.0) \quad 0(0) \quad 0(0) \quad 3(25)$

FIGURE 4 Treatment outcomes of multidrug-resistant tuberculosis patients after exclusion of pre-extensively drug-resistant tuberculosis (pre-XDR-tuberculosis). Data in the bars are treatment success rates. Data below the graphics are presented as $\mathrm{n}(\%)$. Patients in the optimised group were classified into two subgroups, pyrazinamide-susceptible (PZA-S) and pyrazinamide-resistant (PZA-R). Treatment outcomes of patients after exclusion of pre-XDR-TB are shown based on any evidence (a) and susceptibility testing to second-line drugs regardless of the previous treatment experience (b). 
- Cure Completion $\square$ Loss to follow-up

- Lack of conversion at the end of the intensive phase

Adverse drug reactions

Failure

Bacteriological reversion

a)

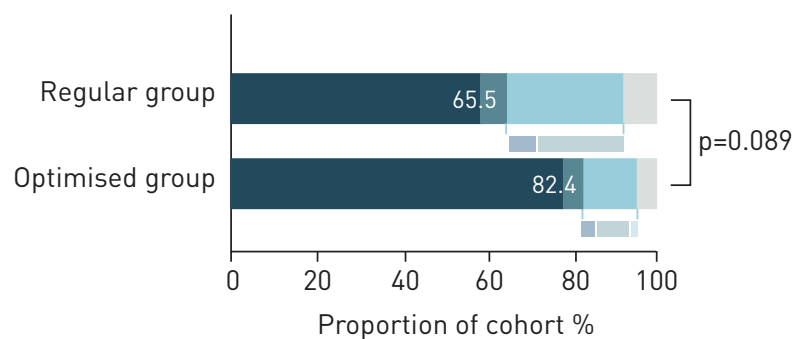

Proportion of cohort \%

PZA-S subgroup

Regular group $(n=29)$

Optimised group $(n=51)$

$17(58.6) \quad 2(6.9) \quad 8(27.6) \quad 2(6.9)$

Cure Completion Failure follow-up b)

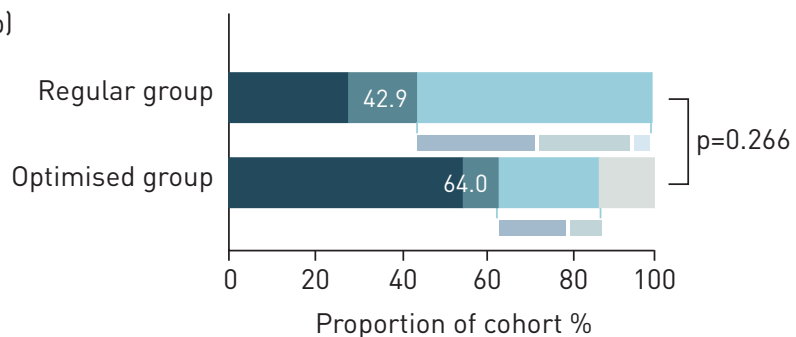

Loss to

Cure Completion Failure follow-up

PZA-R subgroup

Regular group ( $n=21) \quad 6(28.6) \quad 3(14.3) \quad 12(57.1) \quad 0(0)$

Optimised group ( $n=25)$

$\begin{array}{cccc}6(28.6) & 3(14.3) & 12(57.1) & 0(0) \\ 14(56.0) & 2(8.0) & 6(24.0) & 3(12.0)\end{array}$

FIGURE 5 Treatment outcomes of multidrug-resistant tuberculosis patients grouped by pyrazinamide (PZA) susceptibility. Data in the bars are treatment success rates. Data below the graphics are presented as $\mathrm{n}(\%)$. Patients in the regular group were classified into pyrazinamide-susceptible (PZA-S) (a) and pyrazinamide-resistant (PZA-R) (b) subgroups, and treatment outcomes were compared for the corresponding subgroup in the optimised group.

curve was similar in patients with PZA susceptibility whether they were treated with the optimised regimen or the regular regimen (hazard ratio $0.99,95 \%$ CI $0.97-1.01$; figure $6 \mathrm{a}$ ). Nevertheless, the time to conversion decreased dramatically when patients with PZA-R MDR-TB received the optimised regimen (hazard ratio 0.46, 95\% CI 0.22-0.99; figure 6b).

\section{Adverse drug reactions}

Adverse drug reactions were commonly observed during the lengthy treatment process (table 3). More than $70 \%$ of patients reported at least one adverse drug reaction. The total frequency of adverse drug reactions was higher in the regular treatment group than the optimised group $(p=0.013)$. Most side-effect profiles were comparable between the two groups, while nausea and vomiting occurred in a significantly higher proportion of patients in the regular group.

Treatment interruption due to adverse events was infrequent in the optimised group (six out of 76 patients (7.9\%)). In three cases treatment was discontinued because of intractable vomiting and in the other three
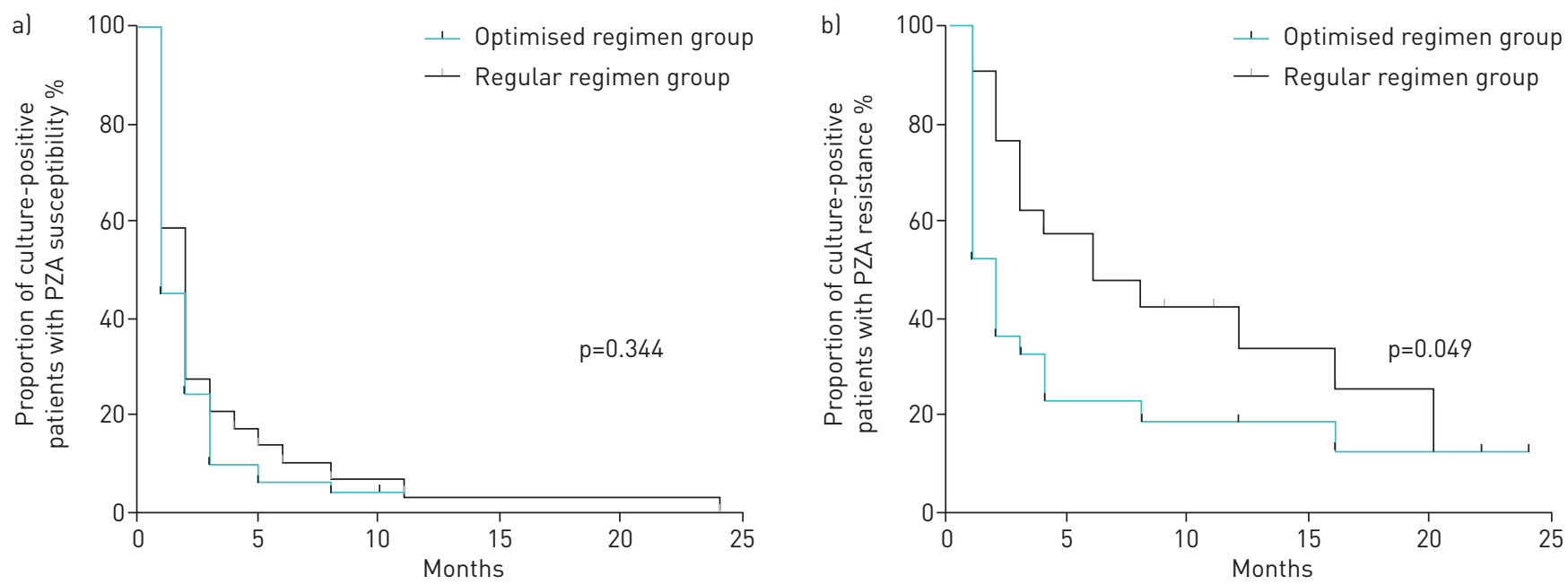

FIGURE 6 Proportion of pyrazinamide (PZA) susceptible (a) and PZA resistant (b) patients with positive sputum cultures and time to conversion. 
TABLE 3 Reported adverse drug reactions during treatment for multidrug-resistant tuberculosis

Reaction

Regimen

Regular (n=76) Optimised PZA-R (n=25)

Optimised PZA-S (n=51)

$\begin{array}{lc}\text { Nausea and vomiting } & 20(26.4) \\ \text { Liver injury } & 17(18.4) \\ \text { Renal injury } & 2(2.6) \\ \text { Arthralgia } & 16(21.1) \\ \text { Hyperuricaemia } & 5(6.6) \\ \text { Thrombocytopenia } & 2(2.6) \\ \text { Leukopenia } & 2(2.6) \\ \text { Anaemia } & 2(2.6) \\ \text { Low-potassium } & 25(30.3) \\ \text { Hypothyroidism } & 10(16.2) \\ \text { Hearing impairment } & 10(16.2) \\ \text { Visual impairment } & 2(2.6) \\ \text { Rash } & 6(7.9) \\ \text { QT interval elongation } & 0(0) \\ \text { Insomnia } & 2(2.6) \\ \text { Epilepsy } & 2(2.6) \\ \text { Peripheral nerve defect } & 4(5.3)\end{array}$

$\begin{array}{cc}4(16.0) & 6(11.9) \\ 4(16.0) & 15(29.4) \\ 0(0) & 1(2.0) \\ 1(4.0) & 6(11.9) \\ 1(4.0) & 12(23.5) \\ 0(0) & 0(0) \\ 1(4.0) & 1(2.0) \\ 0(0) & 0(0) \\ 4(16.0) & 5(9.8) \\ 3(12.0) & 6(11.9) \\ 3(12.0) & 5(9.8) \\ 0(0) & 0(0) \\ 1(4.0) & 1(2.0) \\ 0(0) & 1(2.0) \\ 1(4.0) & 0(0) \\ 0(0) & 0(0) \\ 1(4.0) & 1(2.0)\end{array}$

Data is presented as $n(\%)$. PZA-R: pyrazinamide-resistant; PZA-S: pyrazinamide-susceptible.

cases patients suffered from severe liver injury. In contrast, 12 patients in the regular group reported severe adverse drug reactions leading to treatment failure. Among these patients, four developed severe liver injury, four developed refractory hypokalaemia, two developed epilepsy and two developed hearing impairments.

\section{Discussion}

In this prospective cohort study we demonstrated that a therapy of 12 months duration, as guided by molecular DST for PZA, could improve the outcome of MDR-TB treatment compared with the conventional WHO-approved 24-month regimen (based on both the WHO-outcome definitions and our own study definitions). To our knowledge, this is the first prospective study using molecular DST for PZA to guide MDR-TB treatment that has led to a successful shortening of treatment duration for PZA-S MDR-TB from 24 months to 12 months without use of novel drugs. This finding supports the precision medicine based "individualised approach" to more effective treatment of MDR-TB rather than the program based approach.

Although PZA resistance has been reported as an independent factor associated with poor MDR-TB treatment outcomes in retrospective analysis [31,34], it is important that we demonstrate this in our prospective study. As resistance to fluoroquinolone or SLIDs could affect treatment outcomes in MDR-TB patients, we excluded pre-XDR-TB in subgroup analysis either by history or by confirmed DST results. Nevertheless, the optimised group still achieved better outcomes than the regular group. Several reasons could account for these observations. First, we identified early the PZA-R MDR-TB patients who tended to fail to convert to sputum negative status and replaced PZA with high-dose isoniazid accordingly. Consequently, the anti-tubercular activity of the regimen might be improved, as reflected by a more rapid culture conversion (figure 6) and a decreased treatment failure rate. Secondly, the 12-month treatment regimen (6PZA-Amk-Lfx-Pto-Clr/6PZA-Lfx-Pto-Clr) administered for PZA-S patients in the optimised group could achieve a $82.4 \%$ treatment success rate without relapse after 1 year, while the 24-month regimen (6PZA-Amk-Lfx-Pto-Clr/18PZA-Lfx-Pto-Clr) administered in the regular group achieved a 75.9\% treatment success rate after 1 year of treatment and a $65.5 \%$ treatment success rate at the end of treatment. The comparable 1-year treatment success rates had concordant similar culture conversion curves, which could be explained by the same anti-tubercular regimen being used whether the regimen was guided by PZA resistance information or not. However, during the second year of the regular 24-month treatment subgroup, two patients defaulted and one patient interrupted treatment due to liver injury. We noted that all defaults occurred during the second year for the regular subgroup, while the 12-month regimen subgroup had lower treatment failure rates and default rates, especially for treatment failure due to adverse drug reactions. It can be inferred that for PZA-S MDR-TB, patients were more likely to continue through 
the 12-month treatment without higher relapse risk, even though we did not add any other drugs. Based on our study, it appears that for the PZA-S MDR-TB, 12 months may be long enough to achieve the desired treatment outcome.

Currently, the 9-12 month standardised shorter regimen recommended by the WHO is suitable for MDR-TB cases that are not resistant to and have never been treated with second-line drugs. Therefore, it should not be used if there is documented or suspected resistance to one of the drugs comprising the regimen. By reducing the window of resistance, laboratory errors and turn-around times, new molecular testing at the start of treatment will enable patients to be treated with the shorter MDR-TB regimen more readily and safely, thus reducing transmission of MDR-TB further. Molecular DST for fluoroquinolones has been highly recommended before starting the short course treatment owing to its superior accuracy and the drug's pivotal role in MDR-TB treatment. Nevertheless, the role of molecular tests for detection of resistance to PZA may need to be discussed. DST for PZA was not mandatory in the WHO-recommended 9-month regimen and, in the Bangladesh study, PZA DST was not performed routinely; however, a treatment success rate of $87.9 \%$ was reached, slightly higher than that of this study. We noticed that the Bangladesh regimen requires seven drugs, including both PZA and high-dose isoniazid for all patients. It is possible that a more effective drug might cover up the ineffectiveness of PZA for potential PZA-R patients. On the other hand, this could also be because the role of PZA in shortening the therapy is masked when the treatment is given for 9-12 months, but its effect might still be seen if, in the future, the treatment is given for 6 months. It remains to be seen if the effect of PZA can be seen in the Bangladesh regimen if it is shortened to 6 months. In this study, it is worth noting that PZA-S MDR-TB had a very high sputum conversion rate of $94.2 \%$ at 6 months, compared with $76.9 \%$ for PZA-R MDR-TB patients. Future studies are needed to test if the treatment for PZA-S MDR-TB patients could be shortened to 6 months.

Our subgroup analysis showed that for PZA-S MDR-TB patients (pre-XDR-TB and XDR-TB being excluded), a regimen consisting of five drugs (levofloxacin, prothionamide, amikacin, PZA and clarithromycin) could achieve a $86.4 \%$ treatment success rate without 1 -year relapse, which is similar to that of the WHO-recommended 9-12 month Bangladesh regimen (87\%-90\%) [35-37]. This could indicate that, if PZA resistance is well evaluated at treatment start, treatment duration may be shortened to 12 months with just five drugs on the strength of the crucial role of PZA in shortening the treatment duration if DST shows PZA susceptibility $[13,38]$. On the other hand, in accordance with this study, an individual patient data analysis found that the use of PZA was associated with a lower degree of success and higher mortality for PZA-R patients [39]. For these patients, replacing PZA with other effective drugs, such as linezolid, bedaquiline and clofazimine (which are listed as Group A and B drugs for MDR-TB treatment in the WHO Rapid Communication published in August 2018 [16]), we may still be able to achieve a shorter treatment duration. Currently, clinical trials are undertaking new efforts to shorten the duration of treatment whilst using the minimum number of drugs. PZA is considered by many clinical trials (e.g. the endTB, MDR-END and NeXT trials) as an important supportive drug and these regimens are constituted with no more than five drugs. Therefore, DST for each drug (including PZA) appears to be essential in order to ascertain their effectiveness, especially in settings where resistance to PZA is common. It is encouraging that a recent study accurately detected $M$. tuberculosis mutations associated with resistance to isoniazid, fluoroquinolones and aminoglycosides, which holds promise as a rapid point-of-care test to guide therapeutic decisions for TB patients [40]. In the future, with more rapid availability of drug resistance information, we believe that treatment duration may be shortened with satisfactory outcomes for certain patients who are susceptible to key drugs like PZA.

Compared with reports from the United States [41], The Netherlands [24], Belgium [42], Thailand [43] and even China [25] that demonstrated PZA-resistance rates of around 40\% in MDR-TB isolates, the proportion of PZA-resistance in our study was slightly lower at 36.5\% (46 out of 126 patients who underwent DST for PZA (76 in the optimised group and 50 in the regular group)) and may be attributed to the exclusion of XDR-TB here. Therefore, it is suggested that rapid detection of PZA resistance is vital for effective treatment of MDR-TB in a shorter period of 12 months without incurring additional cost for medication and avoiding unnecessary adverse events associated with prolonged drug use (as in the regular regimen for PZA-S MDR-TB).

This study was designed and launched in 2012 following the 2011 updated WHO guidelines which allowed both cycloserine and clarithromycin use. However, cycloserine was not used in China until the end of 2014 due to concerns regarding tolerance, while clarithromycin was routinely used. Thus 36 out of 76 patients in the regular regimen group in our study received cycloserine when it was available, while all patients in the optimised regimen group received clarithromycin. Interestingly, although some of the regular group cases were taking a seemingly "stronger" drug, the optimised regimen group still achieved better outcomes. Indeed, the regular use of clarithromycin for MDR-TB treatment is barely justified by 
current evidence that reports no improvement in treatment success among patients taking macrolides [21, $39,44]$. Additionally, the registered trials evaluating the efficacy of clarithromycin for MDR-TB treatment are scarce, suggesting the absence of further evidence in the recommendation of this drug. However, clarithromycin is known to have synergistic and immunomodulatory properties [45], especially in its interaction with linezolid [46]. With its good tolerability and good safety profile, this makes clarithromycin a possible adjunct drug in difficult-to-treat cases when five active drugs must be ensured [47].

Our study has several limitations. First, retrospective results for molecular DST of PZA were available for 50 out of 76 regular group patients, while the isolates from the remaining 26 patients were unavailable. As such, the sample size of our subgroup analysis based on PZA resistance was limited. Secondly, the regular group cases came from 10 out of 12 participating hospitals while the optimised group cases came from all 12 hospitals, which may lead to bias due to different care, resources and experience. To minimise bias from different hospitals, all were local area TB hospitals that could provide CDC-certified services for $M$. tuberculosis cultures and DST for rifampicin and isoniazid. In addition, all enroled patients received antitubercular drugs from the same batches for free and received the same follow-up plan for free. Additionally, molecular DST for PZA (another key procedure) was performed for all isolates in Huashan Hospital Central Laboratory.

In the recent STREAM trial $78.1 \%$ of patients achieved a favourable outcome, which is not as high as the previous short-course treatment cohorts that have been reported [35]. Since some key drugs for MDR-TB (such as fluoroquinolones) were also used in treatment of other common bacterial infections, such as community-acquired pneumonia (CAP) and infectious diarrhea, resistance could already exist in the MDR-TB. While a standardised treatment regimen has the advantage of being simple and can be applied in a resource-limited setting more readily, it has the drawback of lack of knowledge of resistance to key drugs at the individual level. This can lead to inadequate treatment in individuals that already have resistance to some key drugs and may possibly lead to amplification of resistance. In contrast, individualised regimens are designed according to the drug resistance profile of the organism an individual harbors and therefore fall into the category of precision medicine [48, 49]. As demonstrated in this study, such a precise approach is important. For example, even though only PZA resistance was evaluated to guide treatment, the treatment outcome rate could be improved to $82.4 \%$ in PZA-S MDR-TB: This gives us confidence to believe that individualised regimens guided by molecular DST for PZA or other core drugs will be a promising future direction for treatment of MDR/XDR-TB, especially with the recent development of rapid and affordable whole-genome sequencing for M. tuberculosis strains [50, 51].

In conclusion, a treatment strategy guided by molecular DST for PZA could offer fresh opportunity to improve the treatment success rate of MDR-TB without the use of new drugs, while reducing adverse reactions. For PZA-S MDR-TB patients, we show that the current longer WHO regimen of $20+$ months may be shortened to 12 months without compromising treatment success. This finding may have important implications for improved treatment and care of MDR-TB patients globally. However, further studies are needed to confirm our findings in other countries.

Acknowledgements: We thank all the physicians from the 12 centres that participated in this study for patient enrolment and follow-up. We thank two statisticians, Wei Zhang (Fudan University) and Junyan Zhang (Clinical Epidemiology and Evidence-Based Medicine, Shanghai Medical Association) for assistance in data management and analysis.

Conflict of interest: None declared.

Support statement: This work was supported by National Major Science and Technology Special Project grants (2013ZX10003008-003-001, 2018ZX10722301-003-001). Y. Zhang was supported in part by NIH grant AI099512. Funding information for this article has been deposited with the Crossref Funder Registry.

\section{References}

1 Global Tuberculosis Report 2017. Geneva, World Health Organization, 2017. http://apps.who.int/medicinedocs/ documents/s23360en/s23360en.pdf

2 Falzon D, Schünem ann HJ, Harausz E, et al. World Health Organization treatment guidelines for drug-resistant tuberculosis, 2016 update. Eur Respir J 2017; 49: 1602308.

3 Moodley R, Godec TR. Short-course treatment for multidrug-resistant tuberculosis: the STREAM trials. Eur Respir Rev 2016; 25: 29-35.

4 Dheda K, Gumbo T, Maartens G, et al. The epidemiology, pathogenesis, transmission, diagnosis, and management of multidrug-resistant, extensively drug-resistant, and incurable tuberculosis. Lancet Respir Med 2017; 5: 291-360.

5 Bouton TC, Phillips PPJ, Mitnick CD, et al. An optimized background regimen design to evaluate the contribution of levofloxacin to multidrug-resistant tuberculosis treatment regimens: study protocol for a randomized controlled trial. Trials 2017; 18: 563.

6 Cellamare M, Ventz S, Baudin E, et al. A Bayesian response-adaptive trial in tuberculosis: the endTB trial. Clin Trials 2016; 14: 17-28. 
7 Lange C, Duarte R, Fréchet-Jachym M, et al. Limited benefit of the new shorter multidrug-resistant tuberculosis regimen in Europe. Am J Respir Crit Care Med 2016; 194: 1029-1031.

8 Munoz-Torrico M, Salazar MA, Millán MDJM, et al. Eligibility for the shorter regimen for multidrug-resistant tuberculosis in Mexico. Eur Respir J 2018; 51: 1702267.

9 Lessells RJ, Cooke GS, McGrath N, et al. Impact of point-of-care xpert MTB/RIF on tuberculosis treatment initiation: a cluster-randomized trial. Am J Respir Crit Care Med 2017; 196: 901-910.

10 Zhang Y, Mitchison D, Shi W, et al. Mechanisms of pyrazinamide action and resistance. Microbiol Spectr 2014; 2 MGM2-0023-2013.

11 Van Deun A, Chiang C-Y. Shortened multidrug-resistant tuberculosis regimens overcome low-level fluoroquinolone resistance. Eur Respir J 2017; 49: 1700223.

12 Nahid P, Dorman SE, Alipanah N, et al. Executive summary: official American Thoracic Society/Centers for Disease Control and Prevention/Infectious Diseases Society of America clinical practice guidelines: treatment of drug-susceptible tuberculosis. Clin Infect Dis 2016; 63: e147-e195.

13 Zhang Y, Mitchison D. The curious characteristics of pyrazinamide: a review. Int J Tuberc Lung Dis 2003; 7: 6-21.

14 Kempker RR, Heinrichs MT, Nikolaishvili K, et al. Lung tissue concentrations of pyrazinamide among patients with drug-resistant pulmonary tuberculosis. Antimicrob Agents Chemother 2017; 61: e00226-17.

15 British Thoracic Association. A controlled trial of six months chemotherapy in pulmonary tuberculosis. First report: results during chemotherapy. Br J Dis Chest 1981; 75: 141-153.

16 Rapid communication: key changes to treatment of multidrug- and rifampicin-resistant tuberculosis (MDR/RR-TB). Geneva, World Health Organization, 2018. www.who.int/tb/publications/2018/WHO_RapidCommunicationMDRTB. pdf

17 Chang KC, Yew WW, Zhang Y. Pyrazinamide susceptibility testing in Mycobacterium tuberculosis: a systematic review with meta-analyses. Antimicrob Agents Chemother 2011; 55: 4499-4505.

18 Whitfield MG, Soeters HM, Warren RM, et al. A global perspective on pyrazinamide resistance: systematic review and meta-analysis. PLoS One 2015; 10: e0133869.

19 Daneau G, Gumusboga M, De Rijk P, et al. The majority of patients with multidrug-resistant tuberculosis in Sub-Saharan Africa present a concomitant resistance to pyrazinamide. Int J Mycobacteriology 2016; 5: S46-S47.

20 Fofana MO, Shrestha S, Knight GM, et al. A multistrain mathematical model to investigate the role of pyrazinamide in the emergence of extensively drug-resistant tuberculosis. Antimicrob Agents Chemother 2017; 61: e00498-16.

21 Ahuja SD, Ashkin D, Avendano M, et al. Multidrug resistant pulmonary tuberculosis treatment regimens and patient outcomes: an individual patient data meta-analysis of 9,153 patients. PLoS Med 2012; 9: e1001300.

22 Zhang Y, Chiu Chang K, Leung C-C, et al. "ZS-MDR-TB" versus "ZR-MDR-TB": improving treatment of MDR-TB by identifying pyrazinamide susceptibility. Emerg Microbes Infect 2012; 1: e5.

23 Scorpio A, Zhang Y. Mutations in $p n c A$, a gene encoding pyrazinamidase/nicotinamidase, cause resistance to the antituberculous drug pyrazinamide in tubercle bacillus. Nat Med 1996; 2: 662-667.

24 Simons SO, Van Ingen J, Van Der Laan T, et al. Validation of pncA gene sequencing in combination with the mycobacterial growth indicator tube method to test susceptibility of Mycobacterium tuberculosis to pyrazinamide. J Clin Microbiol 2012; 50: 428-434.

25 Campbell PJ, Morlock GP, Sikes RD, et al. Molecular detection of mutations associated with first- and second-line drug resistance compared with conventional drug susceptibility testing of Mycobacterium tuberculosis. Antimicrob Agents Chemother 2011; 55: 2032-2041.

26 Miotto P, Cabibbe AM, Feuerriegel S, et al. Mycobacterium tuberculosis pyrazinamide resistance determinants: a multicenter study. MBio 2014; 5: e01819-14.

27 Xia Q, Zhao L-L, Li F, et al. Phenotypic and genotypic characterization of pyrazinamide resistance among multidrug-resistant Mycobacterium tuberculosis isolates in Zhejiang, China. Antimicrob Agents Chemother 2015; 59: 1690-1695.

28 Falzon D, Jaramillo E, Schunemann HJ, et al. WHO guidelines for the programmatic management of drug-resistant tuberculosis: 2011 update. Eur Respir J 2011; 38: 516-528.

29 Shi W, Zhang X, Jiang X, et al. Pyrazinamide inhibits trans-translation in Mycobacterium tuberculosis. Science 2010; 333: 1630-1632.

30 Definitions and reporting framework for tuberculosis-2013 revision. Geneva, World Health Organization, 2013. http://apps.who.int/iris/bitstream/handle/10665/79199/9789241505345_eng.pdf;jsessionid=A05DE62B4AB06CF ABBF6B447D10BEA8B?sequence $=1$

31 Zheng X, Ning Z, Drobniewski F, et al. pncA mutations are associated with slower sputum conversion during standard treatment of multidrug-resistant tuberculosis. Int J Antimicrob Agents 2017; 49: 183-188.

32 Aibana $\mathrm{O}$, Bachmaha $\mathrm{M}$, Krasiuk V, et al. Risk factors for poor multidrug-resistant tuberculosis treatment outcomes in Kyiv Oblast, Ukraine. BMC Infect Dis 2017; 17: 129.

33 Lunt M. Selecting an appropriate caliper can be essential for achieving good balance with propensity score matching. Am J Epidemiol 2014; 179: 226-235.

34 Chang KC, Leung CC, Yew WW, et al. Pyrazinamide may improve fluoroquinolone-based treatment of multidrug-resistant tuberculosis. Antimicrob Agents Chemother 2012; 56: 5465-5475.

35 Van Deun A, Maug AKJ, Salim MAH, et al. Short, highly effective, and inexpensive standardized treatment of multidrug-resistant tuberculosis. Am J Respir Crit Care Med 2010; 182: 684-692.

36 Piubello A, Harouna SH, Souleymane MB, et al. High cure rate with standardised short-course multidrug-resistant tuberculosis treatment in Niger: no relapses. Int J Tuberc Lung Dis 2014; 18: 1188-1194.

37 Kuaban C, Noeske J, Rieder HL, et al. High effectiveness of a 12-month regimen for MDR-TB patients in Cameroon. Int J Tuberc Lung Dis 2015; 19: 517-524.

38 Mitchison DA. The action of antituberculosis drugs in short course chemotherapy. Tubercle 1985; 66: 219-225.

39 Ahmad N, Ahuja SD, Akkerman OW, et al. Treatment correlates of successful outcomes in pulmonary multidrug-resistant tuberculosis: an individual patient data meta-analysis. Lancet 2018; 392: 821-834.

40 Xie YL, Chakravorty S, Armstrong DT, et al. Evaluation of a rapid molecular drug-susceptibility test for tuberculosis. N Engl J Med 2017; 377: 1043-1054. 
41 Chan ED, Laurel V, Strand MJ, et al. Treatment and outcome analysis of 205 patients with multidrug-resistant tuberculosis. Am J Respir Crit Care Med 2004; 169: 1103-1109.

42 Stoffels K, Mathys V, Fauville-Dufaux M, et al. Systematic analysis of pyrazinamide-resistant spontaneous mutants and clinical isolates of Mycobacterium tuberculosis. Antimicrob Agents Chemother 2012; 56: 5186-5193.

43 Jonmalung J, Prammananan T, Leechawengwongs $\mathrm{M}$, et al. Surveillance of pyrazinamide susceptibility among multidrug-resistant Mycobacterium tuberculosis isolates from Siriraj Hospital, Thailand. BMC Microbiol 2010; 10: 223.

44 Fox GJ, Benedetti A, Cox $\mathrm{H}$, et al. Group 5 drugs for multidrug-resistant tuberculosis: individual patient data meta-analysis. Eur Respir J 2017; 49: 1600993.

45 van der Paardt AF, Wilffert B, Akkerman OW, et al. Evaluation of macrolides for possible use against multidrug-resistant Mycobacterium tuberculosis. Eur Respir J 2015; 46: 444-455.

46 Bolhuis MS, Van Altena R, Van Soolingen D, et al. Clarithromycin increases linezolid exposure in multidrug-resistant tuberculosis patients. Eur Respir J 2013; 42: 1614-1621.

47 Van der Paardt AFL, Akkerman OW, Gualano G, et al. Safety and tolerability of clarithromycin in the treatment of multidrug-resistant tuberculosis. Eur Respir J 2017; 49: 1601612.

48 Cox H, Hughes J, Black J, et al. Precision medicine for drug-resistant tuberculosis in high-burden countries: is individualised treatment desirable and feasible? Lancet Infect Dis 2018; 18: 282-287.

49 Migliori GB, Sotgiu G, Rosales-Klintz S, et al. ERS/ECDC statement: European Union standards for tuberculosis care, 2017 update. Eur Respir J 2018; 51: 1702678.

50 Miotto P, Tessema B, Tagliani E, et al. A standardised method for interpreting the association between mutations and phenotypic drug resistance in Mycobacterium tuberculosis. Eur Respir J 2017; 50: 1701354.

51 Cabibbe AM, Walker TM, Niemann S, et al. Whole genome sequencing of Mycobacterium tuberculosis. Eur Respir J 2018; 52: 1801163 . 